$03,09,12$

\title{
Управление циркулярной поляризацией электролюминесценции в спиновых светоизлучающих диодах на основе гетероструктур InGaAs/GaAs $/ \delta\langle\mathrm{Mn}\rangle$
}

\author{
(C) Е.И. Малышева, М.В. Дорохин, П.Б. Дёмина, А.В. Здоровейщев, \\ А.В. Рыков, М.В. Ведь, Ю.А. Данилов
}

Научно-исследовательский физико-технический институт Нижегородского государственного университета им. Н.И. Лобачевского, Нижний Новгород, Россия

E-mail: malysheva@phys.unn.ru

Проведено исследование циркулярно-поляризованной люминесценции светоизлучающих структур InGaAs/GaAs с дельта-легированным слоем Mn в GaAs барьере. Выполнено варьирование параметров структур: однородное и дельта-легирование акцепторной примесью, удаление донорного легирования из технологического процесса. Получено, что величина и знак степени циркулярной поляризации люминесценции существенно зависят от выбранного технологического режима. Одномерное моделирование волновых функций структур выявило хорошее согласие между параметрами циркулярно-поляризованной люминесценции и пространственным распределением волновых функций тяжелых дырок относительно дельта-слоя Мn.

Работа выполнена в рамках реализации государственного задания. Проект № 8.1751.2017/ПЧ Минобрнауки России, при поддержке РФФИ (гранты № 15-02-07824_а, 16-07-01102_a), гранта (МК-8221.2016.2) Президента Российской Федерации.

DOI: 10.21883/FTT.2017.11.45051.18k

\section{1. Введение}

Полупроводниковые структуры с ферромагнитным слоем и активной областью, расположенными на небольшом удалении друг от друга (до $10 \mathrm{~nm}$ ) являются интересным объектом исследования и имеют ряд прикладных применений. В подобных структурах реализуется фундаментальное физическое явление ориентации по спину носителей заряда в активной области за счет обменного взаимодействия с близкорасположенным ферромагнитным слоем (эффект близости) [1-3]. К практическим преимушествам эффекта близости можно отнести возможность создания спиновой поляризации в полупроводнике без использования явления спиновой инжекции, в то время как большинство полупроводниковых гетероструктур ферромагнетик / полупроводник используют именно принцип спиновой инжекции для генерации спин-ориентированных носителей $[4,5]$. Одной из разновидностей структур, в которых проявляется эффект близости, является квантовая гетероструктуpa InGaAs/GaAs с дельта-слоем Mn, встроенным в GaAs барьер на небольшом удалении от квантовой ямы InGaAs [3,6,7]. Ранее было показано, что намагниченный ферромагнитный $\delta\langle\mathrm{Mn}\rangle$-слой обеспечивает спиновую поляризацию носителей в квантовой яме InGaAs. B режиме люминесценции спин-поляризованные носители рекомбинируют с испусканием циркулярнополяризованного света [6,7]. В [8] был предложен механизм спиновой поляризации дырок в квантовой яме, связанный с обменным взаимодействием с ионами Mn в $\delta\langle\mathrm{Mn}\rangle$-слое. В работе [7] на основании полученных экспериментальных результатов была выдвинута гипотеза, что параметр обменного взаимодействия для структур $\mathrm{InGaAs} / \mathrm{GaAs} / \delta\langle\mathrm{Mn}\rangle$ не является постоянной величиной, а варьируется в зависимости от электронной конфигурации атома $\mathrm{Mn}_{\mathrm{Ga}}$ в дельта-слое. Экспериментальным подтверждением такой гипотезы служило обнаруженное изменение знака и степени циркулярной поляризации при варьировании различных технологических параметров структур.

В настоящей работе выполнен дальнейший анализ механизмов спиновой поляризации и циркулярнополяризованной люминесценции в гетероструктурах $\mathrm{InGaAs} / \mathrm{GaAs} / \delta\langle\mathrm{Mn}\rangle$. Для управления зарядовым состоянием атомов $\mathrm{Mn}$ в $\mathrm{GaAs}$ выполнено легирование слоев $\mathrm{GaAs}$ барьера донорными и акцепторными примесями. Рассмотрено три технологических способа введения примеси: легирование буферного слоя атомами $\mathrm{Si}$, дельталегирование GaAs-барьера атомами углерода и его однородное легирование атомами цинка. Показано, что введение примеси позволяет управлять распределением волновой функции дырок между слоями структуры. Распределение волновой функции дырок связывается с экспериментально измеренными значениями степени циркулярной поляризации люминесцентного излучения образцов.

\section{2. Методика эксперимента}

Структуры сформированы комбинированным методом МОС-гидридной эпитаксии (МОСГЭ) в сочетании с импульсным лазерным осаждением (ИЛО) [6-8]. Было 
Параметры исследованных структур GaAs/InGaAs/GaAs/ $\delta\langle\mathrm{Mn}\rangle / \mathrm{GaAs}$

\begin{tabular}{|c|c|c|c|c|}
\hline № структуры & $\begin{array}{c}\text { Легирование } \\
\text { буферного слоя }\end{array}$ & $\begin{array}{c}\text { Легирование } \\
\text { покровного слоя }\end{array}$ & $\begin{array}{c}P_{\mathrm{EL}}(\max ) \text { при } B=100 \mathrm{mT} \\
\text { и } T=10 \mathrm{~K}\end{array}$ & $T_{\mathrm{C}}(\mathrm{K})$ \\
\hline A & $n$-GaAs/InGaAs & $i$-GaAs & $-0.027^{*}$ & $40 \pm 5$ \\
\hline B & $\begin{array}{c}n-\mathrm{GaAs} / \delta\langle\mathrm{C}\rangle / 20 \mathrm{~nm} \\
\mathrm{GaAs} / \mathrm{InGaAs}\end{array}$ & $i$-GaAs & 0.042 & $35 \pm 5$ \\
\hline $\mathrm{C}$ & $\begin{array}{c}n \text {-GaAs } / \delta\langle\mathrm{C}\rangle / 3 \mathrm{~nm} \\
\mathrm{GaAs} / \mathrm{InGaAs}\end{array}$ & $i$-GaAs & 0.080 & $50 \pm 5$ \\
\hline $\mathrm{D}$ & $n$-GaAs/InGaAs & $p$-GaAs & 0.025 & $30 \pm 5$ \\
\hline IA & $i$-GaAs/InGaAs & $i$-GaAs & 0.020 & $40 \pm 5$ \\
\hline
\end{tabular}

Примечание. * Для структуры А приведено минимальное значение (максимальное по абсолютной величине).

сформировано два типа структур. Первый тип представляет собой эпитаксиальные гетероструктуры светоизлучающих диодов (СИД) для исследований в режиме электролюминесценции. Структуры сформированы на подложках $n$-GaAs. Последовательно выращивались следующие слои: $n$-GaAs буферный слой, квантовая яма (КЯ) InGaAs, нелегированный GaAs (толщиной $\left.d_{s}=4 \mathrm{~nm}\right)$. На следующем этапе при пониженной температуре $400^{\circ} \mathrm{C}$ были выращены дельта-слой $\mathrm{Mn}$ и покровный слой $\mathrm{GaAs}$ методом импульсного лазерного осаждения. Содержание $\mathrm{Mn}$ в $\delta$-слое составило $\sim 6.3 \cdot 10^{13} \mathrm{~cm}^{-2}$. Рост при температуре $400^{\circ} \mathrm{C}$ сопровождается диффузией атомов $\mathrm{Mn}$ в слои GaAs, т. е. уширением профиля легирования. В результате реальная ширина дельта-слоя $\mathrm{Mn}$ на половине от максимальной концентрации составляет $\sim 3 \mathrm{~nm}$ [6-8]. Структура А не содержала дополнительных слоев, легированных акцепторами (за исключением $\delta\langle\mathrm{Mn}\rangle$-слоя). В структурах В, $\mathrm{C}$ и D осуществлялось легирование акцепторной примесью. В структурах В и С легирование осуществлялось путем введения дельта-слоя углерода в процессе выращивания буферного слоя GaAs (перед формированием InGaAs квантовой ямы). Расстояние между $\delta\langle\mathrm{C}\rangle$-слоем и КЯ составило $20 \mathrm{~nm}$ (структура В) и $3 \mathrm{~nm}$ (структура С). Слоевая концентрация атомов в дельта- $\langle\mathrm{C}\rangle$-слое составила $2.5 \cdot 10^{12} \mathrm{~cm}^{-2}$. Также формировались структуры с легированием покровного слоя $\mathrm{GaAs}$ атомами $\mathrm{Zn}$ (структуры D). В этом случае покровный слой $\mathrm{GaAs}$ формировался распылением мишени GaAs : Zn. Оценочная концентрация акцепторов в покровном слое составила $\sim 5 \cdot 10^{18} \mathrm{~cm}^{-3}$. Второй тип представляет структура, не содержащая легированных донорной примесью слоев. Удаление легирования донорами из технологического процесса выполнялось с целью изменения изгиба зон в приповерхностных слоях образца. Указанная структура (структура IA) была сформирована на подложке $i$-GaAs. Технология формирования структуры и толщины слоев в точности совпадали с режимами, использованными для структуры А, за исключением того, что буферный слой GaAs в структуре IA специально не легировался. Согласно оценкам, нелегированный слой GaAs имеет фоновую концентрацию дырок, равную $\sim 3-6 \cdot 10^{15} \mathrm{~cm}^{-3}$.
Схемы образцов для исследований представлены на рис. 1, $a$, параметры структур приведены в таблице.

На образцах для исследования электролюминесценции (ЭЛ) (A-D) были сформированы $\mathrm{Au}$ контакты методом электронно-лучевого испарения в вакууме. Методами фотолитографии и химического травления изготавливали меза-структуры диаметром $500 \mu \mathrm{m}$. Контакт к $n$-GaAs подложке формировался искровым вжиганием Sn-фольги. Таким образом формировались светоизлучающие диоды аналогично [6-8]. Для исследований электролюминесценции на образцы подавалось прямое смещение (на Аu-контакт подавался положительный потенциал по отношению к потенциалу подложки). Возбуждаемое ЭЛ излучение регистрировалось со стороны подложки (рис. 1,a). На структуре IА выполнены исследования фотолюминесценции (ФЛ). При исследованиях ФЛ поверхность образца облучалась $\mathrm{He}-\mathrm{Ne}$ лазером мощностью $16 \mathrm{~mW}$. Возбуждаемое ФЛ излучение регистрировалось со стороны подложки (рис. 1, $a$ ).

На сформированных структурах были исследованы магнитополевые зависимости степени циркулярной поляризации фото- и электролюминесценции. Магнитное поле было направлено перпендикулярно поверхности структур. Значение степени циркулярной поляризации рассчитывалось по формуле

$$
P_{\mathrm{EL}, \mathrm{PL}}=\frac{I\left(\sigma^{+}\right)-I\left(\sigma^{-}\right)}{I_{\mathrm{EL}, \mathrm{PL}}},
$$

где $P_{\mathrm{EL}}\left(P_{\mathrm{PL}}\right)-$ степень циркулярной поляризации электро-(фото)люминесценции, $E_{\mathrm{EL}, \mathrm{PL}}-$ интенсивность ЭЛ(ФЛ); $I\left(\sigma^{+}\right), I\left(\sigma^{-}\right)$- интенсивности компонент люминесценции, циркулярно-поляризованных по левому и по правому кругу соответственно. Интенсивности получены интегрированием участка спектра, соответствующего излучательным переходам в InGaAs квантовой яме. Измерения выполнены в диапазоне температур 10-90 K.

Исследования, выполненные ранее [7,9], позволили однозначно показать, что для одной и той же структуры значения степени циркулярной поляризации фото- и электролюминесценции совпадают в пределах погрешности измерений

$$
P_{\mathrm{EL}}=P_{\mathrm{PL}}
$$




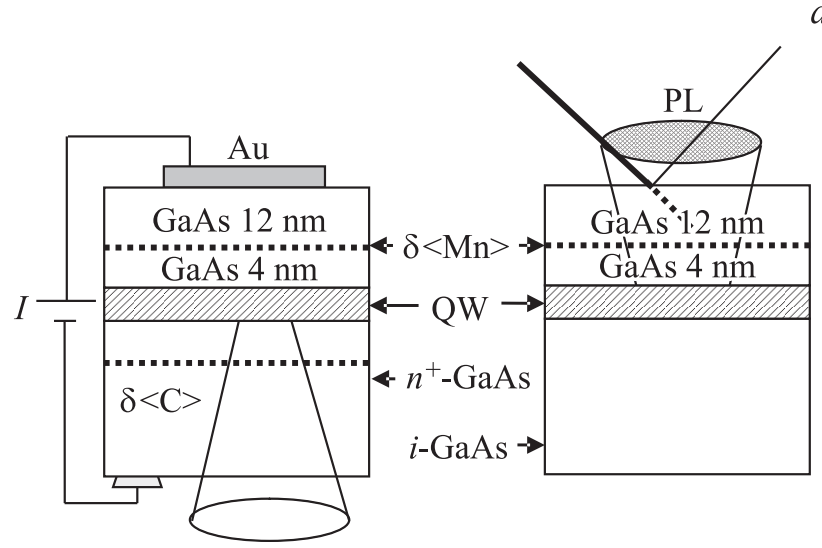

EL
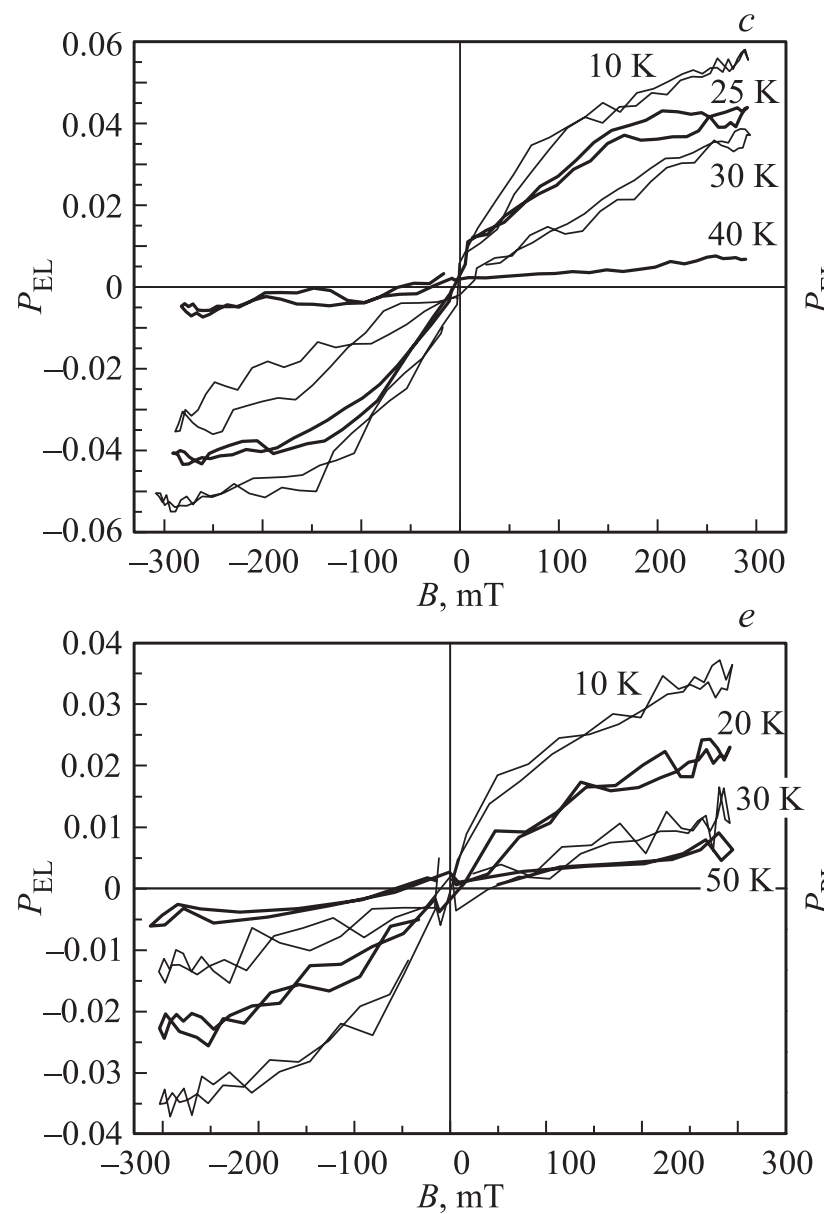
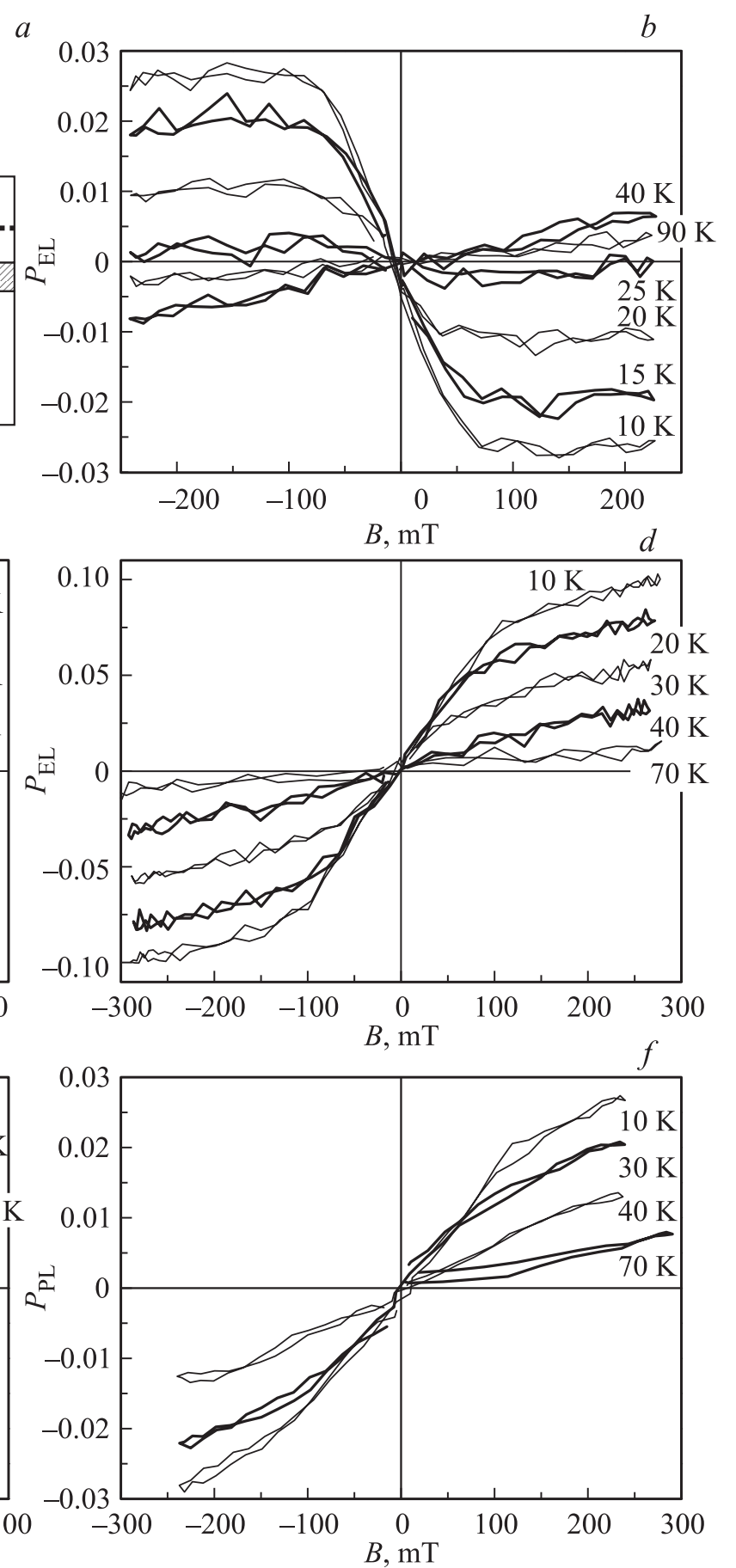

Рис. 1. (a). Схемы исследованных структур: слева - светоизлучающий диод $\delta\langle\mathrm{Mn}\rangle \mathrm{GaAs} / \mathrm{InGaAs}$, сформированный на подложке $n$-GaAs, справа - структура IA, сформированная на подложке $i$-GaAs; $(b-f)$ Магнитополевые зависимости степени циркулярной поляризации люминесценции для структур $\mathrm{InGaAs} / \mathrm{GaAs} / \delta\langle\mathrm{Mn}\rangle$, измеренные при токе диода $5 \mathrm{~mA}$ (для ЭЛ) или мощности накачки $16 \mathrm{~mW}$ (для ФЛ), в диапазоне температур 10-90 K. (b) - структура А-ЭЛ; $(c)$ - структура В-ЭЛ; $(d)$ - структура С-ЭЛ; $(e)$ - структура D-ЭЛ; $(f)$ - структура I-ФЛ.

Равенство (2) является следствием подобия механизмов циркулярной поляризации для фото- и электролюминесценции. В частности, циркулярная поляризация не связана с инжекцией спин-поляризованных носителей из ферромагнитного слоя, а обусловлена поляризацией по спину носителей в активной области. Равенство (2) также позволяет выбирать наиболее удобный способ измерения циркулярной поляризации (ФЛ или ЭЛ) для каждой структуры, а результаты измерений могут быть достоверно сопоставлены друг с другом.

Для анализа механизмов спиновой поляризации носителей в квантовой яме было выполнено одномерное 
моделирование зонной диаграммы и распределения волновых функций дырок в системе $\delta\langle\mathrm{Mn}\rangle / \mathrm{GaAs} / \mathrm{InGaAs}$ в направлении, перпендикулярном плоскости квантовой ямы. Моделирование выполнялось с применением расчетной программы 1DPoisson [10]. При задании параметров программы учитывалось диффузионное размытие ионов $\mathrm{Mn}$, а также уменьшение концентрации дырок вследствие эффекта автокомпенсации.

\section{3. Экспериментальные результаты и обсуждение}

На рис. $1, b-f$ представлены магнитополевые зависимости степени циркулярной поляризации люминесценции для всех исследованных структур, измеренные в диапазоне температур $10-70 \mathrm{~K}$. Зависимость степени циркулярной поляризации от магнитного поля $\left(P_{\mathrm{EL}, \mathrm{PL}}(B)\right)$ при низкой температуре подобна для всех структур, за исключением существенного различия в знаке $P_{\mathrm{EL}, \mathrm{PL}}$. Согласно соотношению (1) положительный знак степени циркулярной поляризации соответствует ситуации, при которой интенсивность поляризованной по левому кругу компоненты превышает интенсивность компоненты, поляризованной по правому кругу (т.е. $\left.I\left(\sigma^{+}\right)>I\left(\sigma^{-}\right)\right)$. Отрицательный знак поляризации соответствует обратной ситуации $\left(I\left(\sigma^{-}\right)>I\left(\sigma^{+}\right)\right)$.

На рассмотренных зависимостях $P_{\mathrm{EL}}(B)$ наблюдается два участка: участок быстрого изменения $P_{\text {EL }}$ в диапазоне магнитных полей 0-0.1 Т (в положительную или отрицательную сторону - рис. $1,(b)$ и $(c-f)$ соответственно), и участок „медленного“ увеличения степени поляризации в полях $0.1-0.3 \mathrm{~T}$. В указанном диапазоне магнитных полей магнитополевая зависимость степени поляризации может быть аппроксимирована линейной функцией, а наклон линейной зависимости практически не различается для разных структур, в том числе и для структур с „отрицательной“ циркулярной поляризацией. Значение $P_{\text {EL }}$ с достаточной степенью точности может быть представлено в виде суммы двух слагаемых

$$
P_{\mathrm{EL}}=P_{\mathrm{EL}}^{\mathrm{FM}}+P_{\mathrm{EL}}^{\mathrm{Z}},
$$

где $P_{\mathrm{EL}}^{\mathrm{FM}}-$ „ферромагнитное“ слагаемое, обусловливающее знак $P_{\mathrm{EL}}$ и быстрое изменение $P_{\mathrm{EL}}$ в низком магнитном поле, $P_{\mathrm{EL}}^{Z}$ - „парамагнитное“ слагаемое. Значение $P$ EL существенно зависит от параметров структур. В магнитном поле $\sim 100 \mathrm{mT}$ значение $P_{\mathrm{EL}}^{\mathrm{FM}}$ выходит на насыщение, и дальнейшее изменение степени поляризации с ростом магнитного поля близко к линейному и связано с „парамагнитным“ слагаемым $P_{\mathrm{EL}}^{Z}$, величина которого слабо зависит от технологических параметров структур, а знак всегда положительный.

При увеличении температуры измерений значение степени циркулярной поляризации на участке быстрого роста $(P$ EL $)$ уменьшается по абсолютной величине и при температуре 30-50 K (в зависимости от параметров структур) становится равным нулю. В этой точке и для более высоких температур на зависимости $P_{\mathrm{EL}}(B)$ имеет место монотонное увеличение степени циркулярной поляризации с ростом магнитного поля, которое может быть описано линейной функцией и которое, по-видимому, соответствует $P_{\mathrm{EL}}^{Z}$ (для представленных примеров такая ситуация соответствует наибольшим температурам измерений). Значение температуры $T=30-50 \mathrm{~K}$, для которой зависимость $P_{\mathrm{EL}}(B)$ преобразуется в линейную, хорошо согласуется с данными по температуре Кюри ферромагнитного $\delta\langle\mathrm{Mn}\rangle$-слоя, полученными в [11].

Для структуры с „отрицательной“ поляризацией (A) характер зависимости $P_{\mathrm{EL}}(B, T)$ более сложный: по мере уменьшения по модулю компоненты $P_{\mathrm{EL}}^{\mathrm{FM}}$ эта величина становится сравнимой с $P_{\mathrm{EL}}^{Z}$, которая практически не зависит от температуры ниже точки Кюри. Это проявляется в смене знака суммарного значения $P_{\mathrm{EL}}$ в меныших магнитных полях. При температуре $40 \mathrm{~K}$ отрицательного значения $P_{\mathrm{EL}}$ для структуры А не зарегистрировано, что соответствует ситуации $P_{\mathrm{EL}}^{Z}>\left|P_{\mathrm{EL}}^{\mathrm{FM}}\right|$. Дальнейшее повышение температуры приводит к увеличению степени циркулярной поляризации, максимальное значение $P_{\mathrm{EL}}$ зарегистрировано при температуре, соответствующей точке Кюри $(50 \mathrm{~K})$, когда отрицательная часть (т.е. $P_{\mathrm{EL}}^{Z}$ ) обращается в нуль. Таким образом, представленные на рис. 2 магнитополевые зависимости степени циркулярной поляризации ЭЛ и ФЛ позволяют установить знак спиновой поляризации носителей в квантовой яме, а также оценить значение температуры Кюри (как минимальной температуры, при которой зависимость $P_{\mathrm{EL}, \mathrm{PL}}(B)$ может быть аппроксимирована линейной функцией [6-9]).

Согласно ранее выполненному анализу, циркулярная поляризация люминесценции исследованных структур связывается со спиновой поляризацией дырок в квантовой яме, которая, в свою очередь, обусловлена обменным взаимодействием с ионами $\mathrm{Mn}$ в близкорасположенном ферромагнитном слое. Вид гамильтониана обменного взаимодействия дырок и ионов $\mathrm{Mn}$ обсуждался в $[8,12,13]$, общее выражение может быть записано как

$$
\hat{H}_{h-\mathrm{Mn}}=N_{0} \beta \sum \int\left(\mathbf{j} \mathbf{S}_{n}\right)|\psi(\mathbf{r})|^{2} \delta\left(\mathbf{r}-\mathbf{R}_{n}\right) d^{3} r,
$$

где $N_{0} \beta$ - параметр обменного взаимодействия, $\mathbf{S}_{n}-$ спиновый оператор иона $\mathrm{Mn}$ в позиции $R_{n}, \mathrm{j}-$ угловой момент дырки, а $\psi, r$ - волновая функция и координата дырок, локализованных в квантовой яме.

Согласно [7], знак спиновой поляризации дырок в исследованной системе определяется константой $N_{0} \beta$. Наблюдаемое в эксперименте изменение знака циркулярной поляризации (и соответствующее изменение знака $\left.P_{\mathrm{EL}}\right)$ при варьировании параметров структур (рис. 1) объясняется изменением знака $N_{0} \beta$. Возможность варьирования константы обменного взаимодействия в структурах (Ga, Mn)As была продемонстрирована в работе [12]. В цитированной работе было показано, что знак константы $N_{0} \beta$ зависит от электронной конфигурации атомов Mn. Согласно [12], положительный знак $N_{0} \beta$ 

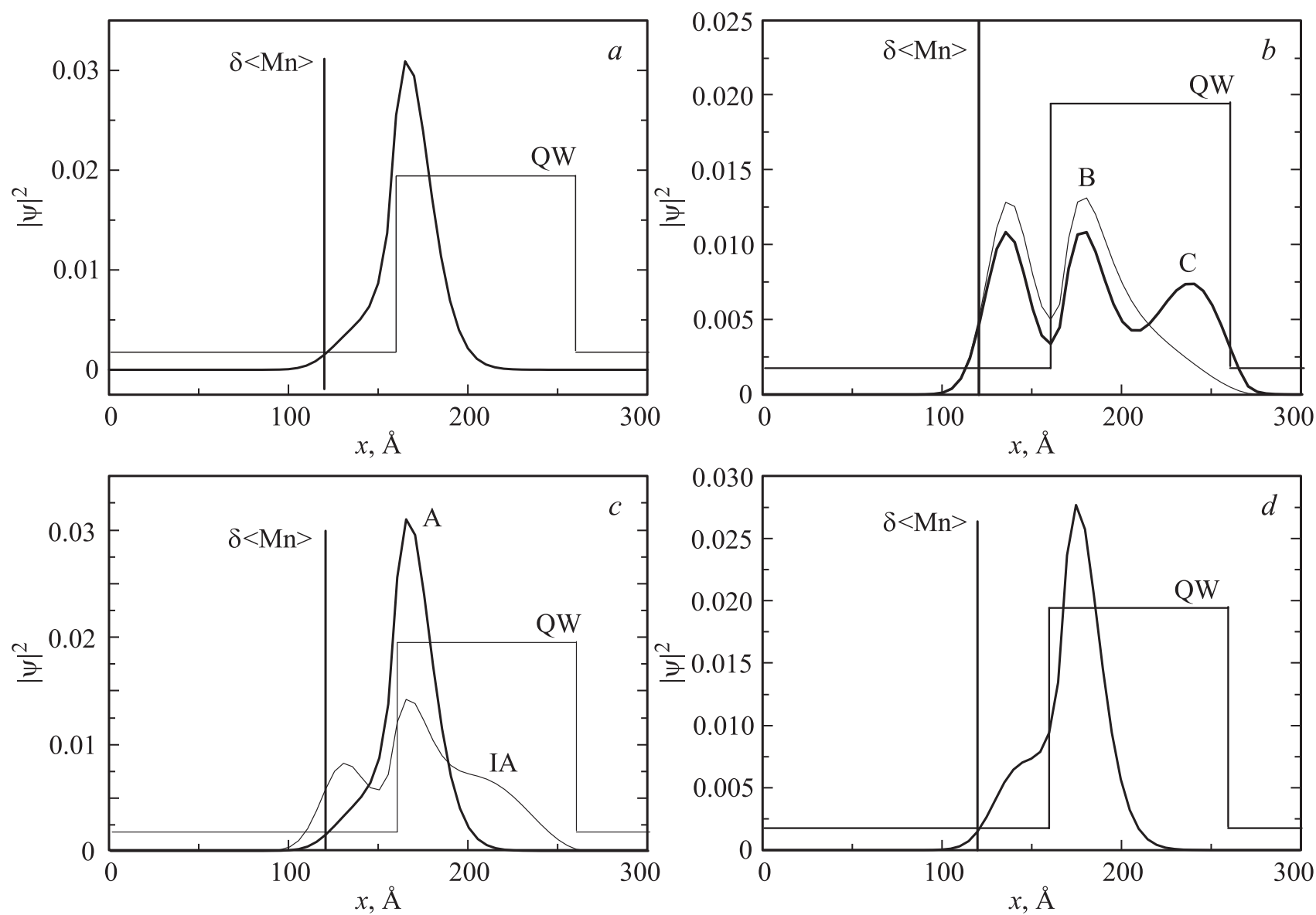

Рис. 2. Рассчитанное по программе [10] одномерное распределение волновых функций тяжелых дырок для структур $\mathrm{InGaAs} / \mathrm{GaAs} / \delta\langle\mathrm{Mn}\rangle$ в направлении, перпендикулярном плоскости квантовой ямы. $(a)$ - структура А светоизлучающего диода, $(b)$ - структура В (кривая В) и структура С (кривая С) с дополнительными $\delta\langle\mathrm{C}\rangle$-легированием, $(c)-$ структура $\mathrm{D}$ с легированием покровного слоя атомами $\mathrm{Zn},(d)$ - структура IA без дополнительно легированных слоев. Жирной вертикальной линией на всех графиках обозначено положение дельта-слоя $\mathrm{Mn}(\delta\langle\mathrm{Mn}\rangle)$, узкими горизонтальными и вертикальными линиями - положение квантовой ямы $(\mathrm{QW})$.

характерен для взаимодействия дырок с нейтральными акцепторами $A^{0}$, отрицательный - для взаимодействия с ионизованными акцепторами $A^{-}$. В реальной структуре могут присутствовать примесные атомы Mn как в нейтральном, так и в ионизованном положении. Рассчитанная в экспериментах величина $N_{0} \beta$ является, как правило, усредненной по большому количеству примесных атомов [12]. Таким образом, изменение знака спиновой поляризации дырок и $P_{\mathrm{EL}}$ мы связываем именно с варьированием знака $N_{0} \beta$. Общепринятой конфигурацией $\mathrm{Mn}$ в $\mathrm{GaAs}$ является $A_{0}\left(3 d^{5}+\right.$ дырка), такая конфигурация обеспечивает ферромагнитное упорядочение между ионами $\mathrm{Mn}$ и носителями [14-16]. Такая „обычная“ конфигурация, вероятно, обусловливает „положительный“ знак поляризации. Эффект изменения знака поляризации связан с изменением состояния $\mathrm{Mn}$ на $A^{-}$, что обусловливает антиферромагнитное упорядочение и отрицательный знак $P_{\mathrm{EL}}$. Указанный эффект предсказан в теоретических работах [14-16], мотивированных экспериментальными результатами для объемных слоев (Ga, Mn)As [12].
В исследованных структурах электронная конфигурация атомов Mn в GaAs также, как и пространственное распределение дырок, варьируются от структуры к структуре в зависимости от технологических параметров. Кроме того, концентрация дырок, как правило, на порядок ниже концентрации атомов марганца в положении $\mathrm{Ga}\left(\mathrm{Mn}_{\mathrm{Ga}}\right)$ в $\delta\langle\mathrm{Mn}\rangle$-слое [17], следовательно, концентрация ионизованных атомов $\mathrm{Mn}$ в области $\delta$-слоя достаточно высока. Для оценки пространственного распределения волновых функций дырок в исследованных структурах были выполнены расчеты зонной диаграммы и энергетических уровней с помощью программы 1DPoisson [10]. Результаты расчетов представлены на рис. 2.

Для структуры А (рис. 2,a) максимум волновой функции дырок смещен в область квантовой ямы, а доля волновой функции и концентрация дырок в области дельтаслоя Mn, согласно расчетам, сравнительно невысока. Вероятно, низкая концентрация дырок не позволяет компенсировать отрицательно заряженные ионы Mn, т. е. наиболее вероятной конфигурацией атомов $\mathrm{Mn}_{\mathrm{Ga}}$ явля- 
ется $A^{-}$. Такая конфигурация может быть обусловлена компенсацией акцепторных уровней $\mathrm{Mn}_{\mathrm{Ga}}$ состояниями $\mathrm{Mn}_{\mathrm{I}}$ (атомы $\mathrm{Mn}$ в положении междоузлия) или другими дефектами низкотемпературного покровного слоя GaAs $[18,19]$. Картине, представленной на рис. 2, a, можно поставить в соответствие „отрицательный“ знак степени циркулярной поляризации для структуры А (рис. $1, b)$, что согласно модели [7] соответствует взаимодействию дырок с ионизованными атомами $\operatorname{Mn}\left(A^{-}\right)$.

В структурах с дополнительным легированием акцепторами (B, C и D) волновая функция тяжелых дырок имеет два максимума: в области квантовой ямы и вблизи центра $\delta\langle\mathrm{Mn}\rangle$-слоя. Доля волновой функции и концентрация дырок в области $\delta\langle\mathrm{Mn}\rangle$-слоя для указанных структур выше, чем для структуры А, а концентрация ионизованных атомов $\mathrm{Mn}_{\mathrm{Ga}}$ ниже. Значения $P_{\mathrm{EL}}$ для структур $\mathrm{B}, \mathrm{C}$ и D ,положительные“: знак степени циркулярной поляризации $P_{\mathrm{EL}}>0$ при $B>0$. Это является согласованным результатом: за счет более высокой общей концентрации дырок и смещения волновой функции в сторону дельта-слоя $\mathrm{Mn}$, преимущественной конфигурацией $\mathrm{Mn}_{\mathrm{Ga}}$ в структурах $\mathrm{B}, \mathrm{C}$ и $\mathrm{D}$ является $A_{0}\left(3 d^{5}+\right.$ дырка), такой конфигурации соответствует именно положительный знак $P_{\text {EL }}$. Отметим, что наибольшее значение степени циркулярной поляризации и температуры Кюри было получено для структуры С, в которой, согласно расчетам, волновая функция дырок имеет наибольшую из всех структур амплитуду в области $\delta\langle\mathrm{Mn}\rangle$-слоя. Указанный результат является согласованным, т. к. повышение концентрации дырок в области GaAs: Mn увеличивает намагниченность и температуру Кюри $[18,19]$.

Рассмотрим график, полученный для структуры IA (рис. $2, d)$. Различие между структурой А и IА заключается лишь в легировании буферного слоя и подложки, причем в структуре А буферный слой легирован донорной примесью. Однако использование нелегированного буферного слоя меняет распределение электрических полей в структуре и волновой функции дырок. В результате для структуры IA (в отличие от структуры А) волновая функция дырок также имеет локальный максимум в области дельта-слоя. Полученный результат также хорошо согласуется с результатами измерения степени циркулярной поляризации люминесценции: для структуры IA получена положительная циркулярная поляризация, величина $P_{\mathrm{PL}}$ сравнительно невелика.

\section{4. Заключение}

Таким образом, установлена взаимосвязь между экспериментально измеренными значениями степени циркулярной поляризации электролюминесценции, температурой Кюри и теоретически рассчитанными распределениями волновых функций дырок в структурах $\delta\langle\mathrm{Mn}\rangle / \mathrm{GaAs} / \mathrm{InGaAs}$, отличающихся легированием слоев GaAs в приповерхностной области. В структурах, для которых получена наибольшая доля волновой функции дырок в области $\delta\langle\mathrm{Mn}\rangle$-слоя, показано наибольшее значение степени циркулярной поляризации люминесценции и температуры Кюри. Для диодной структуры, сформированной на подложке $n$-GaAs, для которой доля волновой функции дырок в области примесных атомов $\mathrm{Mn}$ минимальна, получено отрицательное значение степени циркулярной поляризации электролюминесценции. Полученный результат хорошо согласуется с моделью $[7,12]$, согласно которой знак обменного взаимодействия между дырками в квантовой яме и ионами Mn в дельта-слое зависит от зарядового состояния $\mathrm{Mn}$.

\section{Список литературы}

[1] R.C. Myers, A.C. Gossard, D.D. Awschalom. Phys. Rev. B 69, 161305(R) (2004)

[2] V.L. Korenev, M. Salewski, I.A. Akimov, V.F. Sapega, L. Langer, I.V. Kalitukha, J. Debus, R.I. Dzhioev, D.R. Yakovlev, D. Müller, C. Schröder, H. Hövel, G. Karczewski, M. Wiater, T. Wojtowicz, Yu.G. Kusrayev, M. Bayer. Nature Phys. 12, 85 (2016).

[3] V.L. Korenev, I.A. Akimov, S.V. Zaitsev, V.F. Sapega, L. Langer, D.R. Yakovlev, Yu.A. Danilov, M. Bayer. Nature Commun. 3, 959 (2012).

[4] I. Zutic, J. Fabian, S. Das Sarma. Rev. Mod. Phys, 76, 323 (2004).

[5] M. Holub, P. Bhattacharya. J. Phys. D 40, R179 (2007).

[6] M.A.G. Balanta, M.J.S.P. Brasil, F. Iikawa, U.C. Mendes, J.A. Brum, Yu.A. Danilov, M.V. Dorokhin, O.V. Vikhrova, B.N. Zvonkov. Nature Sci. Rep. 6, 24537 (2016).

[7] M.V. Dorokhin, Yu.A. Danilov, B.N. Zvonkov, M.A. Gonzalez Balanta, M.J.S.P. Brasil, F. Iikawa, U.C. Mendes, J.A. Brum, P.B. Demina, E.I. Malysheva, A.V. Zdoroveischev, A.V. Kudrin. Appl. Phys. Lett. 107, 4, 028531 (2015).

[8] С.В. Зайцев, М.В. Дорохин, А.С. Бричкин, О.В. Вихрова, Ю.А. Данилов, Б.Н. Звонков, В.Д. Кулаковский. Письма в ЖЭТФ 90, 10, 730 (2009).

[9] M.V. Dorikhin, Yu.A. Danilov, P.B. Demina, V.D. Kulakovskii, O.V. Vikhrova, S.V. Zaitsev, B.N. Zvonkov. J. Phys. D 41, 24 (2008).

[10] I.-H. Tan, Snider, E.L. Hu. J. Appl. Phys. 68, 3, 4071 (1990). http://www.nd.edu/ gsnider/

[11] О.В. Вихрова, Ю.А. Данилов, М.В. Дорохин, Б.Н. Звонков, И.Л. Калентьева, А.В. Кудрин. ПЖТФ 35, 14, 8 (2009).

[12] T. Hartmann, S. Ye, P.J. Klar, W. Heimbrodt, M. Lampalzer, W. Stolz, T. Kurz, A. Loidl, H.-A. Krug von Nedda, D. Wolverson, J.J. Davies, H. Overhof. Phys. Rev. B 70, 233201 (2004).

[13] A.O. Govorov, A.V. Kalameitsev. Phys. Rev. B 71, 035339 (2005).

[14] R. Chakarvorty, S. Shen, K.J. Yee, T. Wojtowicz, R. Jakiela, A. Barcz, X. Liu, J.K. Furgyna, M. Dobrowolska. Appl. Phys. Lett. 91, 171118 (2007).

[15] K. Ando, H. Saito, K.C. Agarwal, M.C. Debnath, V. Zayets. Phys. Rev. Lett. 100, 067204 (2008).

[16] M. Berciu, R. Chakarvorty, Y.Y. Zhou, M.T. Alam, K. Traudt, R. Jakiela, A. Barcz, T. Wojtowicz, X. Liu, J.K. Furdyna, M. Dobrowolska. Phys. Rev. Lett. 102, 247202 (2009).

[17] А.В. Рыков, М.В. Дорохин, Е.И. Малышева, П.Б. Демина, О.В. Вихрова, А.В. Здоровейщев. ФТП 50, 1, 3 (2016).

[18] K.M. Yu, W. Walukiewicz, T. Wojtowicz, I. Kuryliszyn, X. Liu, Y. Sasaki, J.K. Furdyna. Phys. Rev. B 65, 201303 (2002).

[19] T. Jungwirth, J. Sinova, J. Masek, J. Kucera, A.H. MacDonald. Rev. Mod. Phys. 78, 822 (2006). 